\title{
Maximización del índice vida útil/volumen de los filtros usados en accionadores de máquinas eléctricas
}

\author{
Maximizing the Operational Life/Volume Index in Filters \\ Used in Adjustable Speed Drives
}

\author{
*Calleja-Gjumlich J.H. \\ Departamento de electrónica \\ Centro Nacional de Investigación y Desarrollo Tecnológico \\ (CENIDET) \\ E-mail:hcalleja@cenidet.edu.mx \\ Guerrero-Ramírez G.V. \\ Departamento de electrónica \\ Centro Nacional de Investigación y Desarrollo Tecnológico \\ (CENIDET) \\ E-mial:gerardogr@cenidet.edu.mx
}

\author{
Jiménez-Grajales H.R. \\ Gerencia de Energías no Convencionales \\ División de Ingenierías Alternas \\ Instituto de Investigaciones Eléctricas (IIE) \\ E-mail:hjimenez@iie.org.mx
}

Información del artículo: recibido: septiembre de 2009, aceptado: agosto de 2010

\section{Resumen}

La confiabilidad es un tópico de capital importancia en los accionadores de máquinas eléctricas. Durante mucho tiempo se ha supuesto que el mayor porcentaje de las fallas se debe al capacitor electrolítico, parte del filtro pasabajas conectado en el enlace de CD. Una estrategia popular para mejorar la confiabilidad consiste en sobredimensionar el componente, especificándolo a tensiones superiores. El inconveniente de este enfoque es que normalmente se obtienen aparatos más voluminosos. En este artículo se propone el empleo del cociente "Vida Útil/Volumen" como índice para evaluar la bondad de un diseño. El índice puede maximizarse si se sigue una metodología basada en el diseño de experimentos; un ejemplo, desarrollado para un accionador a $5 \mathrm{~kW}$, muestra que la mejoría ocurre cuando se disminuye la frecuencia de corte del filtro.

\section{Descriptores}

- confiabilidad

- accionadores de máquinas eléctricas

- vida útil

- volumen

- capacitores

- inductores

* Autor corresponsal 


\begin{abstract}
Reliability is a main issue in standard adjustable-speed drives. The electrolytic capacitor, part of the low-pass filter in the DC-link, has been blamed as a failureprone component, and several approaches have been followed to improve the reliability. A popular strategy to improve the operational life consists in derating the capacitor, to higher nominal voltages. The drawback of the approach, however, is that it produces larger apparatuses. In this paper, the ratio "Operational Lifel Volume" is proposed as an index to evaluate a particular design. The index can be maximized following a methodology based on the design of experiments technique. An example developed for a $5 \mathrm{~kW}$ motor drive shows that the index can be improved by designing the filter at lower cut-off frequencies.
\end{abstract}

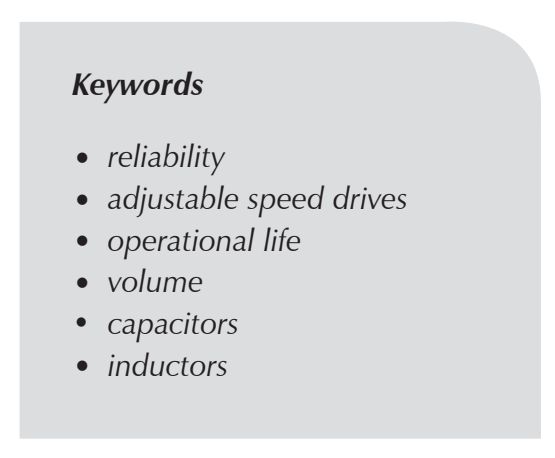

\section{Introducción}

En la figura 1 se muestra el diagrama de un accionador de máquinas eléctricas con un rectificador no controlado como etapa de entrada, un inversor trifásico como etapa de salida y un filtro LC pasa-bajas en el enlace de $\mathrm{CD}$. Una fuente de preocupación es el capacitor electrolítico: la corriente a través de él incluye armónicas producidas tanto por el rectificador como por el inversor. La temperatura interna aumenta, debido al flujo de corriente sobre la resistencia equivalente en serie (ESR), lo que degrada las características del capacitor y acorta la vida útil (Sarjeant et al., 2001). Para alargarla se han seguido diferentes enfoques. Uno consiste en reducir el valor del capacitor a valores tan pequeños que sea posible utilizar elementos de película o de cerámica. Si bien la viabilidad ha quedado demostrada, el enfoque exige el empleo de un rectificador activo como primera etapa (Chien et al., 1998). De hecho, puede eliminarse completamente el capacitor, aunque a costa de un rizado muy elevado en la tensión en el enlace (Piepenbreier et al., 2004); además de la obvia complejidad por la circuitería extra, estos convertidores son muy sensibles a los transitorios (Klimpner et al., 2004), y los desequilibrios en la tensión trifásica de entrada generan un rizado a frecuencia del doble de la de línea, lo que interfiere con la carga e impone restricciones muy severas en el control (Hwang et al., 2007).

A pesar de las propuestas, en aplicaciones que no demandan operación regenerativa se aprovecha la con-

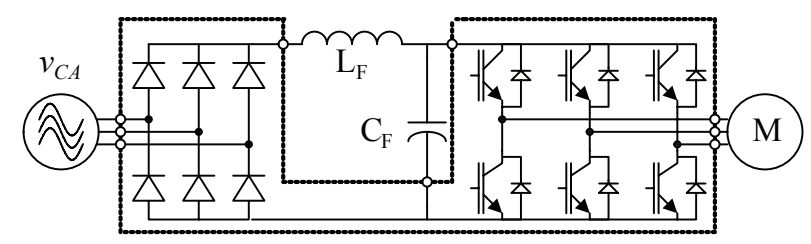

Figura 1. Accionador de máquinas eléctricas con capacitor electrolítico en el enlace de CD figuración de la figura 1 para construir el accionador, porque la confiabilidad de un rectificador sin controlar es muy superior a la de un rectificador activo y las demandas sobre el bloque de control disminuyen sustancialmente. Muchos fabricantes ofrecen módulos integrados que contienen al rectificador no controlado y el inversor trifásico en un mismo empaque (dentro del contorno punteado en la figura 1), los que se dejan sin conectar, a fin de que pueda incluirse un filtro LC entre ellas.

El mayor porcentaje de las fallas se atribuye al capacitor, pero los fabricantes aseguran que en la actualidad los componentes de última tecnología presentan vidas más largas de lo que anteriormente se estimaba (Parler, 2004). Un estudio publicado recientemente parece confirmar este argumento (Hirschmann et al., 2007). Por otro lado, una técnica usual para maximizar la vida útil es el sobredimensionamiento de los componentes (European Space Agency, 2008), aunque este enfoque suele producir un aparato más voluminoso y puede resultar contraproducente en muchas aplicaciones, en las cuales el volumen es un factor parámetro relevante (v.g.: aparatos electrodomésticos).

El proceso de diseño de un filtro LC es bien conocido; sin embargo, no existe una relación explicita entre el comportamiento de las variables eléctricas, la vida útil de los elementos del filtro y el volumen resultante. Como solución a esta dificultad, se presenta aquí un método para relacionar el comportamiento eléctrico del filtro con la vida útil $\left(L_{O P F}\right)$ y el volumen $\left(V_{\text {OLF }}\right)$ resultantes. Los aspectos sobresalientes de la propuesta son:

a) Se propone utilizar el cociente $L_{O P F} / V_{\text {OLF }}$ como índice de valoración del filtro. El índice se expresa en horas de operación por centímetro cúbico (horas $/ \mathrm{cm}^{3}$ ).

b) El método aprovecha la técnica de diseño factorial de experimentos de dos niveles, la que facilita identifi- 
car los parámetros con el mayor impacto sobre el índice de valoración. Una vez identificados los parámetros, el diseño puede mejorarse a fin de cumplir con una meta particular.

La metodología se ilustra con en accionador de $5 \mathrm{~kW}$.

\section{Análisis basado en el diseño de experimentos}

La meta consiste en ponderar los parámetros de diseño de acuerdo al impacto que tienen sobre la vida útil y el volumen. Debido a la cantidad de información involucrada, el proceso de ponderación se complica conforme aumenta el número de parámetros considerados. El proceso se puede simplificar utilizando la técnica de Diseño de Experimentos, la que analiza el efecto simultáneo de varios factores (o parámetros) sobre la variable de salida. Una variante de esta técnica es el diseño factorial, en el que todos los parámetros se modifican a la vez y se llevan a cabo experimentos (o evaluaciones de la variable de salida) para todas las combinaciones de niveles de los parámetros. Este procedimiento revela el efecto de un parámetro cuando los otros están cambiando (Telford, 2007). En un diseño factorial de dos niveles, a cada parámetro se le asigna un valor mínimo y otro máximo, denotados por $L$ y $H$, respectivamente. La variable de salida es el cociente $L_{O P F} / V_{O L F}$ y se consideran los tres siguientes parámetros de diseño $(n=3)$ :

- Frecuencia de corte del filtro $f_{C}$. Debe ser lo suficientemente reducida como para generar un voltaje de CD razonablemente libre de rizo, según lo demande la aplicación. Los valores mínimo y máximo considerados son $f_{C L}=55 \mathrm{~Hz} \mathrm{y} f_{C H}=80 \mathrm{~Hz}$.

- El factor de amortiguamiento del filtro $\xi$. Debe ofrecer una respuesta transitoria adecuada. Los valores mínimo y máximo considerados son $\xi_{L}=0.3$ y $\xi_{H}=$ 0.6 .

- El voltaje nominal del capacitor $V_{R}$. Debe ser superior al voltaje máximo que se espera en el bus de CD. Los valores mínimo y máximo son $V_{R L}=350 \mathrm{~V}$ y $V_{R H}=450 \mathrm{~V}$

El primer paso del análisis consiste en definir una matriz de diseño; en ella, cada renglón corresponde a una combinación de parámetros y a un experimento. Con $n$ $=3 \mathrm{y}$ usando un diseño factorial completo de dos niveles, se tienen ocho combinaciones. Es necesario entonces diseñar el filtro para cada una de las combinaciones y después evaluar el volumen, la vida útil resultante y el cociente entre ellos.

El núcleo de la técnica de diseño de experimentos es el análisis de variancia. Sea $P$ cualquiera de los parámetros de diseño. El efecto principal $\Delta A v g_{P}$, debido a cada parámetro se calcula promediando la diferencia entre las respuestas que se obtienen para cada uno de los niveles del parámetro (O'Connor, 2002); es decir:

$\Delta \operatorname{Avg} g_{P}=\operatorname{Avg}(H)_{P}-\operatorname{Avg}(L)_{P}$

donde:

$$
\begin{aligned}
& \operatorname{Avg}(H)_{P}=\left.\frac{1}{4} \sum_{j=1}^{4} x_{j}\right|_{P=H} \\
& \operatorname{Avg}(L)_{P}=\left.\frac{1}{4} \sum_{j=1}^{4} x_{j}\right|_{P=L}
\end{aligned}
$$

y a su vez, $\left.x_{j}\right|_{P=M}$ y $\left.x_{j}\right|_{P=L}$ son los valores de la variable de salida cuando $P$ toma los valores máximo y mínimo, respectivamente. Además, si $\Delta A v g_{p}$ es positivo, el incrementar el parámetro $P$ ocasionará un incremento en la variable de salida. Con $\Delta A v g_{p}$ negativo, el efecto es el opuesto.

\section{Diseño del filtro}

Aunque sólo incluye dos elementos, el filtro debe satisfacer una serie de requerimientos: que las pérdidas sean mínimas, que la corriente que se demanda al rectificador no sea discontinua, que el rizo de tensión $\Delta v_{D C}$ a su salida sea menor a un valor dado, etc. El enfoque seguido toma en cuenta el factor de amortiguamiento (Rajashekara et al., 1987).

El circuito equivalente se muestra en la figura 2; $R_{F}$ modela las pérdidas en el filtro y $R_{\mathrm{L}}$ modela, de manera simplificada, al motor. La simplificación implica que sólo se considera la potencia promedio demandada por el motor operando en régimen permanente (suponiendo un inversor sin pérdidas). $R_{L}$ depende de la resistencia del rotor y puede aproximarse con:

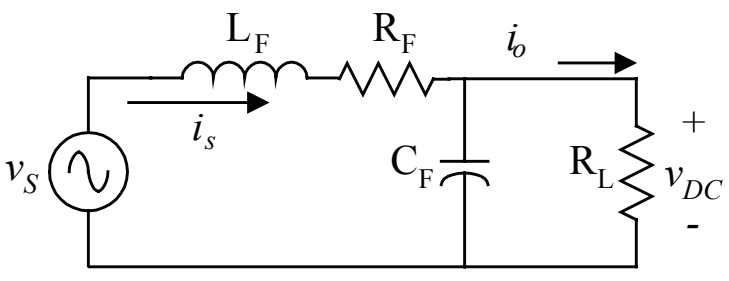

Figura 2. Filtro pasa-bajas LC 
$R_{L}=\frac{V_{N}^{2}}{P_{N}}$

donde $V_{N}$ y $P_{N}$ son el voltaje y la potencia nominales del motor, respectivamente. Por medio de análisis convencional se obtiene la función de transferencia, en términos de la frecuencia natural $\omega_{n}$ y el factor de amortiguamiento $\xi$ :

$g(s)=G_{0} \frac{\omega_{n}{ }^{2}}{s^{2}+2 \xi \omega_{n} s+\omega_{n}{ }^{2}}$

donde:

$G_{O}=\frac{R_{L}}{R_{F}+R_{L}}$

$\omega_{n}=\frac{1}{\sqrt{G_{O} L_{F} C_{F}}}$

$\xi=\sqrt{\frac{G_{O}}{4 L_{F} C_{F}}}\left(R_{F} C_{F}+\frac{L_{F}}{R_{L}}\right)$

Si se especifican $\omega_{n} \mathrm{y} \xi$, los elementos del filtro pueden calcularse resolviendo las siguientes ecuaciones:

$$
\begin{aligned}
& R_{F} C_{F}^{2}+\frac{2 \xi}{G_{O} \omega_{n}} C_{F}+\frac{1}{R_{L} G_{O} \omega_{n}^{2}}=0 \\
& L_{F}=\frac{1}{C_{F} G_{O} \omega_{n}{ }^{2}}
\end{aligned}
$$

\section{Volumen}

El volumen del filtro $V_{\text {OLF }}$ es la suma del volumen del inductor $V_{O L L}$ con el del capacitor $V_{O L C}$.

\section{Inductor}

Un factor básico en el diseño de inductores es el producto de áreas $\mathrm{A}_{\mathrm{p}}$, el cual corresponde al área de la ventana, multiplicada por el área de la sección transversal del núcleo. Para laminación E-I, $A_{\mathrm{P}}$ se relaciona con la energía $W$ que debe manejar el inductor a través de la siguiente relación:
$A_{P}=\left\{\frac{2 W \times 10^{4}}{\mathrm{~K}_{\mathrm{U}} \mathrm{B}_{\mathrm{m}} \mathrm{K}_{\mathrm{J}}}\right\}^{1 / 0.88}$

donde $\mathrm{B}_{\mathrm{m}}$ es la densidad de flujo magnético, la constante $K_{U}$ es el factor de utilización de la ventana y $K_{J}$ depende del incremento de temperatura en el inductor (McLyman, 1988). La energía que debe manejar $L_{F}$ depende de la potencia nominal $P_{O}$ de la carga y de la tensión línea a neutro $V_{L N}$ a la entrada del rectificador:

$W=\frac{\pi^{2} L_{F} P_{O}^{2}}{108 V_{L N}^{2}}$

Usando $\mathrm{K}_{\mathrm{U}}=0.4, \mathrm{~B}_{\mathrm{m}}=1$ Tesla y $\mathrm{K}_{\mathrm{J}}=534$ (para un incremento de temperatura de $50^{\circ} \mathrm{C}$ ):

$A_{P}=\left\{8.556 \frac{P_{O^{2} L F}}{\mathrm{VLN}^{2}}\right\}^{1 / 0.88}$

Por otro lado, el volumen $V_{O L L}$ que ocupa el inductor, depende también de $A_{p}$, de acuerdo a:

$V_{O L L}=K_{V} A^{0.75}$

donde la constante $\mathrm{K}_{\mathrm{V}}$ es igual a 19.7 para laminación E-I. Sustituyendo en la ecuación (14), el volumen del inductor, en función de parámetros eléctricos, queda como:

$V_{O L L}=122.75\left\{\frac{P_{O}^{2} L_{F}}{\mathrm{~V}_{\mathrm{LN}^{2}}}\right\}^{0.75 / 0.88}$

\section{Capacitor}

Por lo que se refiere a capacitores, los fabricantes los ofrecen en tamaños estándar que dependen de la capacitancia y del voltaje nominal. Así pues, al seleccionarse el componente de acuerdo a estos valores se define el volumen $V_{O L C}$ de él, ya que la ficha técnica proporciona el diámetro y la altura.

\section{Vida útil}

Inductor

Los inductores exhiben tasas de fallos muy bajas. Por ejemplo, de acuerdo a datos extraídos del manual militar MIL HDBK 217-F, un inductor en un ambiente típi- 
co de una aplicación industrial, con una temperatura interna de $80^{\circ} \mathrm{C}$, exhibe una tasa de fallos de 0.001 fallas por millón de horas (Department of Defense-EUA, 1991). Por lo tanto, la vida útil del filtro estará limitada por el capacitor.

\section{Capacitor}

La vida útil del capacitor $L_{O P C}$ puede describirse matemáticamente con (Parler, 2004):

$$
L_{O P C}=L_{B} f_{1}(V) f_{2}(\Delta T)
$$

donde $L_{B}$ es la vida útil nominal a la temperatura interna máxima $T_{M^{\prime}}$ y depende del diámetro del capacitor, como se aprecia en la tabla 1.

Tabla 1. Vida útil para capacitores electrolíticos tipo PEH200, a $T_{M}=85^{\circ} \mathrm{C}($ Evox, 2001)

\begin{tabular}{cccccc}
\hline Diámetro $(\mathrm{mm})$ & 35 & 50 & 65 & 75 & 90 \\
\hline$L_{B}\left(10^{3}\right.$ horas $)$ & 20 & 24 & 30 & 40 & 60 \\
\hline
\end{tabular}

Sean $V_{A}$ el voltaje aplicado al capacitor y $V_{R}$ el voltaje máximo permitido. El término $f_{1}$ considera el efecto del esfuerzo del voltaje aplicado al capacitor y está dado por:

$$
f_{1}(V)=4.3-3.3 \frac{V_{A}}{V_{R}}
$$

A su vez, $f_{2}$ es función del incremento en la temperatura interna y se expresa como:

$$
f_{2}(\Delta T)=2^{(T M-T H S) / K}
$$

donde $K$ es una constante que depende de la construcción y $T_{H S}$ es la temperatura interna en la aplicación. El término $T_{H S}$ depende de la potencia $P_{C}$ disipada en el capacitor, de su re-

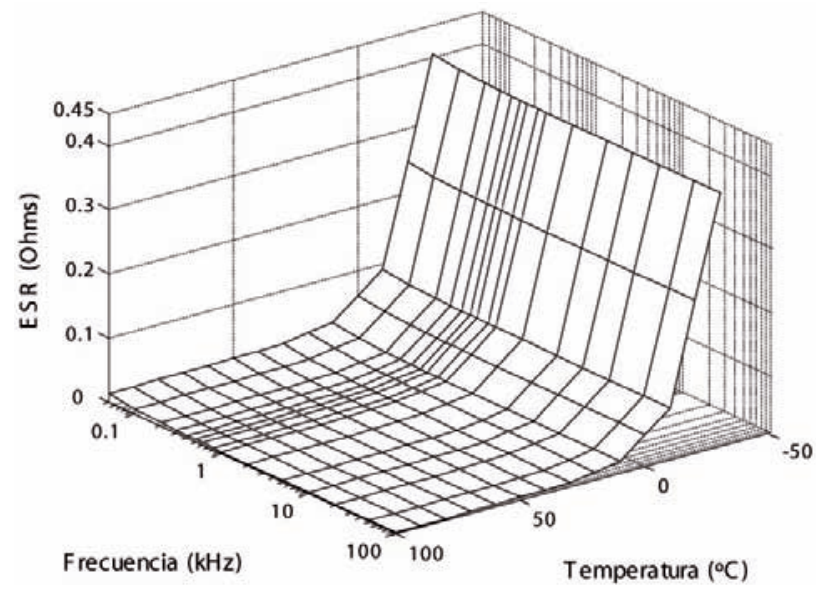

sistencia térmica $R_{\theta}$ y de la temperatura ambiente $T_{A}$, de acuerdo con:

$T_{H S}=T_{A}+R_{\theta} P_{C}$

La potencia $P_{C}$ depende de la ESR y de la corriente $i_{C}$ que fluye a través del capacitor. La ESR exhibe un comportamiento alineal, como puede apreciarse en la figura 3 , la que corresponde a un capacitor electrolítico de $4700 \mu \mathrm{F}$ a $450 \mathrm{~V}$. La ESR también depende, de forma inversamente proporcional, del valor de capacitancia y de $V_{R}$.

El contenido armónico de la corriente $i_{C}$ depende de la tensión trifásica aplicada al rectificador y de la modulación del inversor. Si se conocen las amplitudes de las armónicas $I_{h}$ y los valores $E S R_{h}$ a las mismas frecuencias, la potencia $P_{C}$ se calcula con:

$$
P_{C}=\sum_{h=1}^{j} \operatorname{ESR}_{h} I_{h}^{2}
$$

En este punto, la dificultad reside en el comportamiento de la ESR, función de la frecuencia y de la temperatura. Por lo tanto, para calcular $P_{C}$ es necesario seguir un procedimiento iterativo, suponiendo inicialmente que $T_{H S O}=T_{A^{\prime}}$ y que se conocen tanto las amplitudes armónicas $I_{h}$ como la matriz de ESR del capacitor.

Entonces:

a) Por medio de interpolación, calcular una curva de ESR en función de la frecuencia, para la temperatura $T_{H S O}$.

b) Utilizando nuevamente interpolación, calcular los valores de $E S R_{h}$ a las frecuencias de las armónicas en corriente.

c) Calcular $P_{C}$ utilizando la ecuación (20).

d) Calcular la temperatura $T_{H S}$ utilizando la ecuación (19).

e) Si ITHS - THSO I es mayor a un error e previamente especificado, el proceso se repite haciendo $\mathrm{T}_{\mathrm{HS}}=\mathrm{T}_{\mathrm{HSO}}$.

Una vez calculada la temperatura $T_{H S}$, se procede al cálculo de $L_{O P C}$ utilizando las ecuaciones (16), (17) y (18).

Figura 3: ESR como función de la temperatura $T_{H S}$ y la frecuencia 


\section{Accionador a 5 kW}

El procedimiento se probó con una accionador de $5 \mathrm{~kW}$. Las cuatro columnas a la izquierda de la tabla 2 corresponden a la matriz de diseño. Se obtuvieron 8 diseños del filtro con los datos de la segunda y tercera columnas, haciendo $\omega \mathrm{n}=2 \pi f_{\mathrm{C}}$, y siguiendo el proceso de la sección 3; al resolverse la ecuación (9) se toma la solución que arroja el capacitor más grande. Los volúmenes se calculan de acuerdo a la sección 4. Para calcular $L_{O P C}$ es necesario determinar las magnitudes $I_{h}$ de los armónicos. Para ello, el vector que describe a $i_{C}$ se obtiene por medio de simulación, usando el esquemático de la figura 4 , con una modulación PWM con portadora triangular a $3 \mathrm{kHz}$ y el modelo de una máquina de inducción jaula de ardilla trifásica.

Las magnitudes $I_{h}$ se calculan aplicando la transformada rápida de Fourier al vector de $i_{C}$. Una vez que se determinan estas amplitudes es posible aplicar el procedimiento de la sección 5 , haciendo $L_{O P F}=L_{O P C}$. Las columnas de la derecha, en la tabla 2, corresponden a los resultados.

Los efectos principales de los parámetros calculados de acuerdo a la sección 2, se enlistan en la tabla 3, con fines comparativos. Se enlistan también los efectos principales sobre el volumen y sobre la vida útil.

De acuerdo a las magnitudes y signos de los efectos principales con mayor impacto en las variables de sali$\mathrm{da}$, se tiene lo siguiente.

- Si se desea maximizar la vida útil, es conveniente aumentar $V_{R}$, con ello se obtiene un capacitor de mayor tamaño, el que exhibirá una $L_{B}$ mayor.

- La tabla 3 lista los efectos principales para aumentar el volumen; por lo tanto, si se desea minimizarlo, lo que conviene hacer es reducir $V_{R^{\prime}}$ lo que arrojará un capacitor más pequeño.

- Tanto la vida útil como el volumen dependen del mismo parámetro, pero con sentidos opuestos; sin embargo, el efecto principal con mayor impacto sobre el índice $L_{O P F} / V_{O F L}$ es la frecuencia de corte. Para $V_{R}$ y $\xi$ dados, el usar una frecuencia de corte más baja tiende a producir elementos más grandes lo que, si bien aumenta el volumen, también incrementa la vida útil en magnitud suficiente para tener un efecto neto positivo sobre el índice.

\begin{tabular}{ccccccc}
\hline & & & & & & \\
$\mathrm{m}$ & $f_{n}[\mathrm{~Hz}]$ & $\xi$ & $\mathrm{V}_{\mathrm{R}}[\mathrm{V}]$ & $L_{\text {OPF }}[$ horas $]$ & $V_{\text {OLF }}\left[\mathrm{cm}^{3}\right]$ & $\mathrm{LoPF} /$ VoLF $\left[\right.$ horas $\left./ \mathrm{cm}^{3}\right]$ \\
\hline$(1)$ & $(2)$ & $(3)$ & $(4)$ & $(5)$ & $(6)$ & $(7)$ \\
1 & 55 & 0.3 & 350 & $2.76 \mathrm{E}+06$ & 713 & $3.88 \mathrm{E}+03$ \\
2 & 55 & 0.3 & 450 & $2.82 \mathrm{E}+06$ & 873 & $3.23 \mathrm{E}+03$ \\
3 & 55 & 0.6 & 350 & $2.08 \mathrm{E}+06$ & 901 & $2.31 \mathrm{E}+03$ \\
4 & 55 & 0.6 & 450 & $4.76 \mathrm{E}+06$ & 1660 & $2.87 \mathrm{E}+03$ \\
5 & 80 & 0.3 & 350 & $1.24 \mathrm{E}+06$ & 713 & $1.74 \mathrm{E}+03$ \\
6 & 80 & 0.3 & 450 & $2.54 \mathrm{E}+06$ & 829 & $3.07 \mathrm{E}+03$ \\
7 & 80 & 0.6 & 350 & $1.90 \mathrm{E}+06$ & 682 & $2.79 \mathrm{E}+03$ \\
8 & 80 & 0.6 & 450 & $2.93 \mathrm{E}+06$ & 1146 & $2.55 \mathrm{E}+03$ \\
\hline
\end{tabular}

Tabla 2- Matriz de diseño y resultados

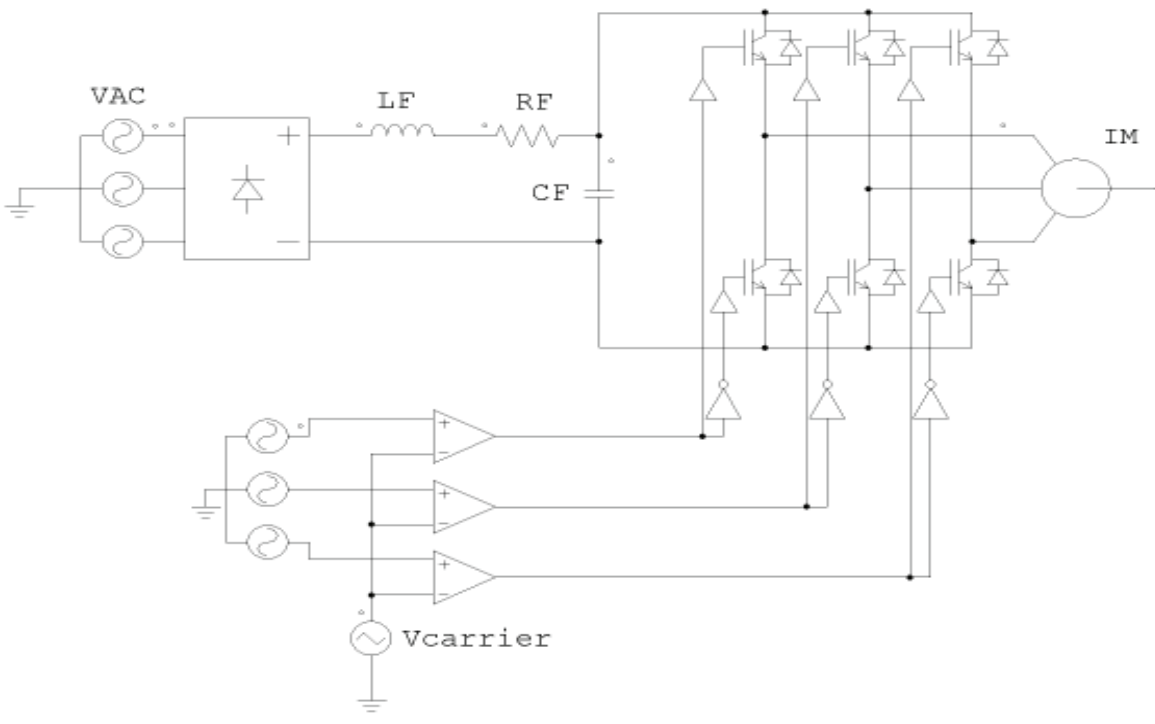

Figura 4. Circuito usado para la simulación 
Tabla 3. Efectos principales de los parámetros de diseño

\begin{tabular}{cccc}
\hline & $\Delta A v g_{f C}$ & $\Delta A v g_{\xi}$ & $\Delta A v g_{V r}$ \\
\hline $\mathrm{L}_{\mathrm{OPF}}[$ horas $]$ & $-9.53 \times 10^{5}$ & $5.75 \times 10^{5}$ & $1.27 \times 10^{6}$ \\
$\mathrm{~V}_{\mathrm{OLF}}\left[\mathrm{cm}^{3}\right]$ & -194.25 & 315.25 & 374.25 \\
LOPF $/$ VoLF $\left[\right.$ horas $\left./ \mathrm{cm}^{3}\right]$ & $-5.32 \times 10^{2}$ & $-3.51 \times 10^{2}$ & $2.49 \times 10^{2}$ \\
\hline
\end{tabular}

El efecto de $f_{C}$ sobre el cociente $L_{O P F} / V_{O F L}$ es evidente en la tabla 2 (compárense las combinaciones 1, 2 y 4 con las 5,6 y 8, respectivamente). Se aprecia también que el cociente para la combinación 3 (a $55 \mathrm{~Hz}$ ) es menor que el de la 7 (a $80 \mathrm{~Hz}$ ). Debe notarse que los volúmenes, tanto de los capacitores como de los inductores, cambien a pasos y no de manera continua en función del valor del elemento. Para el caso de los inductores, una laminación de dimensiones dadas permite construirlos dentro de un intervalo $L_{M A X} \geq L_{F} \geq L_{\text {MIN }}$ y se obtiene una mayor eficiencia volumétrica si $L_{F} \cong L_{M A X}$. Lo mismo ocurre con los capacitores, y el efecto se extiende también a la vida útil nominal $L_{B}$ asociada con las dimensiones del empaque. En el caso de las combinaciones 3 y 7 , para maximizar el cociente $L_{O P F} / V_{O F L}$ puede aprovecharse el efecto principal de $\xi$. Al reducirse este factor se obtiene un capacitor más pequeño, a costa de un inductor más voluminoso; el resultado se aprecia comparando la columna 3 con la 1, o la 4 con la 2.

\section{Conclusiones}

Se presenta un método para maximizar el cociente $L_{P} /$ $V_{O L}$ del filtro LC que se utiliza en accionadores de máquinas eléctricas. El método se basa en el diseño de experimentos y permite identificar qué parámetros de diseño tienen un mayor efecto sobre la vida útil. En este sentido, el método asemeja el análisis de sensitividad, aunque este último no puede aplicarse en casos como el presente, porque no existe una relación explicita entre los parámetros y la variable de salida. De acuerdo con los resultados, para maximizar el cociente $L_{O P F} / V_{O L F}$ conviene minimizar la frecuencia de corte $f_{C}$ (parámetro con el mayor impacto) y el factor de amortiguamiento $\xi$ (parámetro con el segundo mayor impacto).

\section{Referencias}

Chien W., Tzou Y. Analysis and Design on the Reduction of DCLink Electrolytic Capacitor for AC/DC/AC Converter Applied to AC Motor Drives. En: Power Electronics Specialists Confe- rence $\left(29^{\circ}\right.$, 1998. Fukuoka, Japón), Fukuoka, Japón. IEEE, 1998, 275-279.

Department of Defense. Mil. Hdbk 217-F, Reliability Prediction of Electronic Equipment. EUA. 1991.

European Space Agency. Standard ECSS-Q-ST-30-11C. Space Product Assurance. Derating -EEE Components. Space Components Steering Board and European Space Agency. Julio 2008.

Evox Rifa. PEH200 electrolytic capacitors data sheet [en línea]. Disponible en:

http://www.evoxrifa.com/electrolytic_cat/peh200.pdf

Hirschmann D., Tissen D., Schröder S., De Doncker R. Reliability Prediction for Inverters in Hybrid Electrical Vehicles. IEEE Transactions on Power Electronics. 22(6):2511-2517. Noviembre 2007. ISSN: 0885-8993

Hwang G., Lehn P., Winkelnkemper M. Control of Grid Connected AC-DC Converters with Minimized DC Link Capacitance Under Unbalanced Grid Voltage Condition. En: 12ํㅡㄹ European Conference on Power Electronics and Applications Proceedings, Aalborg, Dinamarca, EPE, 2007, pp. 1-10.

Klimpner C., Liserre M. Blaabjerg F. Improved Control of an Active-Front-End Adjustable Speed Drive with a Small DC-Link Capacitor Under Real Grid Conditions. En: 35th Annual IEEE Power Electronics Specialists Conference Record, Aachen, Alemania. IEEE, 2004, pp. 1156-1162.

McLyman-William. Transformer and Inductor Design Handbook. $2^{\circ}$ edición. Nueva York. Marcel Dekker 1988. pp. 145-173.

O'Connor-Patrick. Practical Reliability Engineering. 4a edición. West Sussex, Inglaterra. John Wiley and Sons. 2002. Pp. 247289.

Parler S. Reliability of CDE Aluminum Electrolytic Capacitors. Application Note, Cornell Dubilier Electronics [en línea]. Disponible en: http://www.cde.com/tech/reliability.pdf

Piepenbreier B., Sack L. Regenerative Drive Converter with LineFrequency Switched Rectifier and without DC Link Components. En: 35th Annual IEEE Power Electronics Specialists Conference Record, Aachen, Alemania. IEEE, 2004, pp. 39173923.

Rajashekara K., Rajagopalan V., Sevigny A., Vithayathil J. DC Link Filter Design Considerations in Three-Phase Voltage Source Inverter-Fed Inductor Motor Drive System. IEEE Transactions on Industry Applications. 23(4):673-680, Julio-Agosto 1987. ISSN: 0278-0046.

Sarjeant W., Clelland I., Price R. Capacitive Components for Power Electronics. Proceedings of the IEEE. 89(6): 846-855. Junio 2001, ISSN: 0018-9219.

Telford J. A Brief Introduction to Design of Experiments. Johns Hopkins APL Technical Digest, 27(3): 224-232. Septiembre 2007. ISSN: 0270-5214. 


\section{Semblanza de los autores}

Jorge Hugo Calleja-Gjumlich. Es ingeniero en comunicaciones y electrónica graduado en la Escuela Superior de Ingeniería Mecánica y Eléctrica del Instituto Politécnico Nacional en 1977, obtuvo la maestría en la Facultad de Ingeniería, UNAM en 1981 y el doctorado en el Centro Nacional de Investigación y Desarrollo Tecnológico en el 2000. Colaboró en la Facultad de Ingeniería de la UNAM y en el Departamento de Electrónica del Instituto de Investigaciones Eléctricas. Actualmente es profesor-investigador en el Centro Nacional de Investigación y Desarrollo Tecnológico. Sus líneas de investigación son los sistemas fotovoltaicos interconectados a la red eléctrica y la optimización de convertidores electrónicos de potencia. Es miembro del Sistema Nacional de Investigadores.

Gerardo Vicente Guerrero-Ramírez. Obtuvo el grado de ingeniero industrial electricista por el Instituto Tecnológico de Morelia (1980), el de maestro en ciencias en ingeniería electrónica por el Centro Nacional de Investigación y Desarrollo Tecnológico (1994) y el de doctor en ingeniería en la Facultad de Ingeniería de la UNAM (2001). Actualmente es profesor-investigador en el Centro Nacional de Investigación y Desarrollo Tecnológico. Sus líneas de investigación son el control no lineal de sistemas electromecánicos, con énfasis en los accionadores de máquinas eléctricas enfocados a aplicaciones de tracción.

Humberto Raúl Jiménez-Grajales. Obtuvo el grado de ingeniero industrial en electrónica en el Instituto Tecnológico de Tuxtla (1999) y el de maestro en ciencias en ingeniería electrónica en el Centro Nacional de Investigación y Desarrollo Tecnológico (2002). Actualmente es investigador en la gerencia de energías no convencionales del Instituto de Investigaciones Eléctricas. Sus líneas de investigación son los sistemas fotovoltaicos interconectados a la red eléctrica y la optimización de convertidores electrónicos de potencia. 